\section{Present-day spreading motion of the mid-Atlantic ridge}

\author{
JIN Shuanggen \& ZHU Wenyao \\ Shanghai Astronomical Observatory, Chinese Academy of Sciences, \\ Shanghai 200030, China \\ Correspondence should be addressed to Jin Shuanggen (e-mail: sgjin@ \\ center.shao.ac.cn)
}

\begin{abstract}
Over a long period of time, the spreading rate of the mid-Atlantic ridge has been ascertained by the geological age of the magnetic stripe laid out on either side of the mid-ocean ridge in conjunction with the annals of the magnetic reversal history. Is the mid-Atlantic ridge still spreading today? How fast is it moving or are there any changes? All these questions remain unsolved. Based on the newest ITRF2000 velocity field published by the International Earth Rotation Service (IERS), the contemporary global plate motion model ITRF2000VEL is constructed, independent of any plate model hypothesis. Solutions for relative Euler's vectors of plate pairs as North America-Eurasia, North AmericaAfrica and South America-Africa are obtained, implying current spreading rates of the mid-Atlantic ridge. Comparing them with results from the NNR-NUVEL1A model displays the present-day motion characteristics of the mid-Atlantic ridge that the mid-ridge of the South Atlantic, whose spreading is slowing down, spreads faster than the North Atlantic, whose spreading is speeding up.
\end{abstract}

Keywords: plate motion, Atlantic spreading, ITRF, Euler vector, space geodesy.

With the capacity of date accumulated from oceanic explorations, Hess presented his hypothesis of the origin of the ocean, namely the seafloor spreading theory ${ }^{[1,2]}$. He proposed that the continent and the seafloor drifted passively on a flowing layer of mantle instead of the continent drifting positively on the seafloor as hypothesized in the early continental drifting theory. The seafloor spreading theory was strengthened further when additional oceanic exploring results, such as the seafloor crust tectonics, geomagnetism as well as the distribution of hypocenters and terrestrial heat flows, were obtained during subsequent years. For a long time, people have noticed that some continents on the Earth's surface can fit together like toy bricks. The best case is the remarkable fit along the matching coastlines of South America and Africa. Such a phenomenon demonstrates the existence of divergence of the continental lithosphere as well as the secular drifting of the continental slab, which is largely a consequence of the spreading of the Atlantic Ocean. The spreading of the mid-Atlantic ridge is the basis of the seafloor spreading theory and typical evidence as well for constructing the theory of plate tectonics. For quite some time, the spreading rate of the mid-Atlantic ridge was measured by Ville and Matthews ${ }^{[2]}$, relied on the geological age of the mag- netic stripe laid out on either side of the mid-ocean ridge in conjunction with the annals of the magnetic reversal history. We should point out that such a mid-ocean spreading rate from geological and geophysical data was based on the assumption that the spreading rate was invariant in millions of years. However, geological and geomagnetic evidence of millions of years exhibits that the spreading rate of the mid-Atlantic ridge is variable. Spreading rates of the relevant magnetic strip alter along different transform-fault boundaries, explaining that the Atlantic Ocean spreads erratically. The remarkable diversion of hotspots' track is a very good proof. Therefore, whether or not the mid-Atlantic ridge is still spreading today, how fast it moves or how it changes against the a-million-year average rate, are still unsolved. Those elements, which directly lead to the flux of the human inhabiting environment, should be given full attention so that we can better understand the environmental flux of different time scales through the earth movement history as well as the disasters of various forms.

It is until recent 20 years that space-geodetic techniques like VLBI, SLR, GPS develop rapidly, providing a guarantee for studying the global crustal motion as precise as in millimeters ${ }^{[3,4]}$. In this note, we construct the newest global plate motion model ITRF2000VEL based on the velocity field of 600 global fuducial stations, including the ITRF2000 velocity field free of any plate model hypothesis and IGS velocity field. Our model can better describe the current characteristics of global pate motion. We obtain the spreading rate of the mid-Atlantic ridge from the ITRF2000VEL model and a contrast is performed next with the results predicted by the NNR-NUVEL1A model.

\section{The global plate motion model ITRF2000VEL}

The latest ITRF2000 velocity Field published by the International Earth Rotation Service (IERS) is collected by highly sophisticated techniques, such as VLBI, SLR, GPS and DORIS, including global 360 stations, about 650 results of measurement with different surveying technologies whose precisions are $\pm 1.0 \mathrm{~cm}$ or better. Thereby, it is possible to determine a high-accuracy global plate motion model to study the characteristics ${ }^{[5]}$ of the contemporary plate motion. 241 stations are carefully selected for research by criteria ${ }^{[6,7]}$ as follows: ( $\mathrm{i}$ ) sites on the plate boundaries and seismic zones are rejected; (ii) sites with accuracy $\sigma_{v}>5 \mathrm{~mm} / \mathrm{a}$ are excluded; and (iii) sites established for less than 3 years are excluded.

Of the 3 criteria, the first criterion is the most important one because a misjudgment will lead to an incorrect result. For example, 5 of the 18 sites on the South American plate, which are located on the western edge of the plate, have notable difference in the horizontal movement along the longitude from those plate-interior sites, due to a forceful westward push from the Nazca plate. As 
NOTES

Table 1 A comparison of Euler's vectors of different models

\begin{tabular}{|c|c|c|c|c|c|}
\hline Plate & Model & $\Omega /\left(^{\circ}\right) \cdot \mathrm{Ma}^{-1 \mathrm{a})}$ & $\lambda\left(\left(^{\circ}\right)^{\mathrm{a})}\right.$ & $\varphi /\left(\left(^{\circ}\right)^{\mathrm{a})}\right.$ & $N_{i}^{\text {a) }}$ \\
\hline \multirow{4}{*}{ AFRC } & NNR-NUVEL1A & 0.291 & -74.0 & 50.6 & \\
\hline & ITRF96VEL & $0.275 \pm 0.0028$ & $-84.8 \pm 1.44$ & $51.3 \pm 0.54$ & 12 \\
\hline & ITRF97VEL & $0.274 \pm 0.0020$ & $-86.1 \pm 1.00$ & $53.0 \pm 0.42$ & 13 \\
\hline & ITRF2000VEL & $0.272 \pm 0.0017$ & $-86.4 \pm 1.10$ & $50.3 \pm 0.51$ & 15 \\
\hline \multirow{4}{*}{ ANTA } & NNR-NUVEL1A & 0.238 & -115.8 & 63.0 & \\
\hline & ITRF96VEL & $0.250 \pm 0.002$ & $-138.2 \pm 3.0$ & $57.5 \pm 2.5$ & 8 \\
\hline & ITRF97VEL & $0.255 \pm 0.0008$ & $-135.1 \pm 1.8$ & $62.6 \pm 1.2$ & 8 \\
\hline & ITRF2000VEL & $0.231 \pm 0.0007$ & $-126.9 \pm 1.6$ & $62.3 \pm 1.1$ & 9 \\
\hline \multirow{4}{*}{$\mathrm{ARAB}$} & NNR-NUVEL1A & 0.543 & -4.5 & 45.0 & \\
\hline & ITRF96VEL & 0.580 & -2.4 & 45.84 & 2 \\
\hline & ITRF97VEL & $0.670 \pm 0.068$ & $16.3 \pm 11.0$ & $47.0 \pm 6.0$ & 2 \\
\hline & ITRF2000VEL & $0.611 \pm 0.051$ & $7.44 \pm 7.0$ & $46.5 \pm 4.4$ & 3 \\
\hline \multirow{4}{*}{ AUST } & NNR-NUVEL1A & 0.646 & 33.2 & 33.9 & \\
\hline & ITRF96VEL & $0.636 \pm 0.006$ & $40.2 \pm 0.74$ & $35.0 \pm 0.52$ & 9 \\
\hline & ITRF97VEL & $0.606 \pm 0.004$ & $39.0 \pm 0.50$ & $34.0 \pm 0.42$ & 10 \\
\hline & ITRF2000VEL & $0.622 \pm 0.003$ & $37.9 \pm 0.29$ & $32.5 \pm 0.24$ & 15 \\
\hline \multirow{4}{*}{ CARA } & NNR-NUVEL1A & 0.214 & -93.0 & 25.0 & \\
\hline & ITRF96VEL & 0.216 & -94.3 & 30.3 & \\
\hline & ITRF97VEL & $0.310 \pm 0.140$ & $-85.1 \pm 17.5$ & $34.0 \pm 19.4$ & 4 \\
\hline & ITRF2000VEL & $0.226 \pm 0.120$ & $-92.8 \pm 9.4$ & $27.8 \pm 8.6$ & 5 \\
\hline \multirow{4}{*}{ COCS } & NNR-NUVEL1A & 1.510 & -115.8 & 24.5 & \\
\hline & ITRF96VEL & 1.536 & -114.5 & 24.7 & \\
\hline & ITRF97VEL & 1.544 & -114.6 & 24.6 & \\
\hline & ITRF2000VEL & 1.532 & -115.6 & 24.1 & \\
\hline \multirow{4}{*}{ EURA } & NNR-NUVEL1A & 0.234 & -112.3 & 50.6 & \\
\hline & ITRF96VEL & $0.262 \pm 0.0025$ & $-98.3 \pm 1.0$ & $59.6 \pm 0.34$ & 74 \\
\hline & ITRF97VEL & $0.263 \pm 0.0018$ & $-97.6 \pm 0.68$ & $59.3 \pm 0.28$ & 87 \\
\hline & ITRF2000VEL & $0.258 \pm 0.0010$ & $-99.7 \pm 0.31$ & $57.5 \pm 0.18$ & 98 \\
\hline \multirow{4}{*}{ INDI } & NNR-NUVEL1A & 0.545 & 0.3 & 45.5 & \\
\hline & ITRF96VEL & 0.558 & 3.85 & 47.2 & \\
\hline & ITRF97VEL & 0.550 & 2.93 & 47.1 & \\
\hline & ITRF2000VEL & 0.557 & 3.9 & 53.6 & \\
\hline \multirow{4}{*}{ NAZC } & NNR-NUVEL1A & 0.743 & -100.1 & 47.8 & \\
\hline & ITRF96VEL & 0.764 & -99.8 & 48.9 & \\
\hline & ITRF97VEL & $0.690 \pm 0.093$ & $-100.6 \pm 4.5$ & $36.7 \pm 6.66$ & 2 \\
\hline & ITRF2000VEL & $0.722 \pm 0.083$ & $-97.7 \pm 4.3$ & $44.7 \pm 6.0$ & 2 \\
\hline \multirow{4}{*}{ NOAM } & NNR-NUVEL1A & 0.207 & -85.9 & -2.4 & \\
\hline & ITRF96VEL & $0.200 \pm 0.0022$ & $-84.4 \pm 0.35$ & $-4.4 \pm 0.57$ & 44 \\
\hline & ITRF97VEL & $0.186 \pm 0.0016$ & $-81.95 \pm 0.26$ & $-9.14 \pm 0.46$ & 62 \\
\hline & ITRF2000VEL & $0.197 \pm 0.0010$ & $-83.5 \pm 0.17$ & $-7.33 \pm 0.25$ & 57 \\
\hline \multirow{4}{*}{ PAFC } & NNR-NUVEL1A & 0.641 & 107.3 & -63.0 & \\
\hline & ITRF96VEL & $0.700 \pm 0.0028$ & $91.8 \pm 0.62$ & $-62.4 \pm 0.26$ & 27 \\
\hline & ITRF97VEL & $0.670 \pm 0.0024$ & $94.5 \pm 0.59$ & $-64.0 \pm 0.22$ & 25 \\
\hline & ITRF2000VEL & $0.635 \pm 0.0017$ & $120.3 \pm 0.48$ & $-63.3 \pm 0.20$ & 28 \\
\hline \multirow{4}{*}{ PHIL } & NNR-NUVEL1A & 0.900 & -35.35 & -38.0 & \\
\hline & ITRF96VEL & 0.894 & -33.22 & -34.7 & \\
\hline & ITRF97VEL & 0.896 & -33.38 & -34.6 & \\
\hline & ITRF2000VEL & $0.877 \pm 0.084$ & $-34.52 \pm 4.4$ & $-36.7 \pm 6.1$ & 2 \\
\hline \multirow{4}{*}{ SOAM } & NNR-NUVEL1A & 0.116 & -124.4 & -25.3 & \\
\hline & ITRF96VEL & $0.105 \pm 0.01$ & $-133.6 \pm 9.4$ & $-10.15 \pm 5.3$ & 5 \\
\hline & ITRF97VEL & $0.096 \pm 0.007$ & $-139.4 \pm 4.8$ & $-21.7 \pm 2.5$ & 6 \\
\hline & ITRF2000VEL & $0.109 \pm 0.006$ & $-133.2 \pm 2.6$ & $-19.3 \pm 1.6$ & 7 \\
\hline
\end{tabular}

a) $\Omega, \lambda$ and $\varphi$ represent the rotation velocity, longitude and latitude respectively; $N_{i}$ is the number of sites. 
their movements are not in agreement with the whole plate motion, we need to reject these sites.

According to the theory of tectonic plate motion, if there are more than two stations on a plate, the $\Omega_{i}$ can be estimated through a weighted least squares algorithm $^{[8,9]}$,

$$
V_{i}=\Omega_{i} \times r_{i},
$$

where $\Omega_{i}$ represents the Euler vectors of the " $i$ " plate, " $V_{i}$ " and " $\boldsymbol{r}_{i}$ " represent the velocity and position vector of sites on the " $i$ " plate respectively. We used a weighted least squares algorithm to estimate the Euler vectors of the Africa (AFRC), Antarctica (ANTA), Australia (AUST), Eurasia (EURA), Nazca (NAZC), North American (NOAM), Pacific (PAFC) and South American plate (SOAM) with the velocity field chosen from ITRF2000. The Euler vectors of the INDI, JUFU, ARAB, COCS and PHIL plate cannot be obtained directly because of the lacking or even non-existence of stations. We have to calculate Euler vectors of the 5 plates by using the additive relation of the rotation vectors ${ }^{[10]}$.

$$
\Omega_{i}=\Omega_{i j}+\Omega_{j}
$$

where $\Omega_{i}$ represents one of the Euler vectors of the five plates, and $\Omega_{i j}$ represents the relative Euler vector of adjacent plates. At last, we gain the Euler vectors of global plates (see table 1).

In table 1, the Euler's vectors of ITRF2000VEL, ITRF96VEL ${ }^{[7]}$, ITRF97VEL ${ }^{[7]}$ and NNR-NUVEL1A ${ }^{[9]}$ are presented. As we can see, our estimation precise of Euler vectors is very high due to the high precise of the velocity field. With respect to the ITRF96 and ITRF97, the quantity and quality of ITRF2000 stations have been largely improved and also the distribution is much more extensive. For example, the total number of sites increases from 500 in ITRF97 to 650 in ITRF2000. Thus ITRF2000 can provide a more reasonable and more precise plate motion model than ITRF97 and ITRF96. The global plate motion model of ITRF2000VEL is determined from an about 20-year time span of space geodetic data, so the model can better describe contemporary features of global plate motion than the geological model NNR-NUVEL1 $\mathrm{A}^{[9]}$ that was determined from the geological and geophysical data in several million years. In addition, the ITRF2000VEL model is almost consistent with the geological model NNR-NUVEL1A, but there are some discrepancies of about $10 \%$ in Euler angular velocity and $12^{\circ}$ in Euler pole. These discrepancies are very significant for the contemporary crustal deformation measurement in $\mathrm{mm} / \mathrm{a}$ accuracy. Hence, the ITRF2000VEL model is more suitable as the background of deformation investigation for the current short time scale than the NNR-NUVEL1A.

\section{The present-day spreading rate of the mid-Atlantic ridge}

The Euler's motion formula $V_{i j}=\Omega_{i j} \times \boldsymbol{r}$ is used to determine the current spreading rate of the mid-Atlantic ridge, where $\boldsymbol{r}$ stands for the position vector of the mid-Atlantic ridge, $\Omega_{i j}$ stands for the relative Euler's vector of plate pairs as North America-Eurasia, North America-Africa and South America-Africa derived from space geodetic measurements (table 2), and $V_{i j}$ represents the spreading rate of the mid-Atlantic ridge. In this way, the current spreading rate of the mid-Atlantic ridge are gained, and results are shown in table 3.

Table 3 presents two groups of spreading rates at the North, Equator and South mid-Atlantic ridge. Values predicted by the geological and geomagnetic model NNRNUVEL1A are $21.8 \mathrm{~mm} / \mathrm{a}, 29.8 \mathrm{~mm} / \mathrm{a}$ and $32.9 \mathrm{~mm} / \mathrm{a}$ respectively, and the ones derived from space geodetic data are $22.3 \mathrm{~mm} / \mathrm{a}, 28.8 \mathrm{~mm} / \mathrm{a}$ and $30.7 \mathrm{~mm} / \mathrm{a}$ respectively. We can easily find in fig. 1 that the current spreading rate of the South mid-Atlantic ridge from space geodetic data is slower than that derived from the NNRNUVEL1A model and it is reversed in the North mid-Atlantic ridge.

The NNR-NUVEL1A model is determined from millions of years' geological and geophysical data, so its

\begin{tabular}{|c|c|c|c|c|c|c|}
\hline \multirow{2}{*}{ Plate } & \multicolumn{3}{|c|}{ NNR-NUVEL1A } & \multicolumn{3}{|c|}{ ITRF2000VEL } \\
\hline & $\Omega(x)$ & $\Omega(y)$ & $\Omega(z)$ & $\Omega(x)$ & $\Omega(y)$ & $\Omega(z)$ \\
\hline NOAM & 0.0148 & -0.2063 & -0.0087 & 0.0220 & -0.1932 & -0.0250 \\
\hline SOAM & -0.0593 & -0.0865 & -0.0496 & -0.0704 & -0.0750 & -0.0360 \\
\hline EURA & -0.0564 & -0.1374 & 0.1808 & -0.0234 & -0.1366 & 0.2176 \\
\hline AFRC & 0.0509 & -0.1776 & 0.2249 & 0.0299 & -0.1698 & 0.2077 \\
\hline NOAM-EURA & 0.0712 & -0.0689 & -0.1895 & 0.0454 & -0.0566 & -0.2426 \\
\hline NOAM-AFRC & -0.0361 & -0.0287 & -0.2336 & -0.0079 & -0.0234 & -0.2327 \\
\hline SOAM-AFRC & -0.1102 & 0.0911 & -0.2745 & -0.1003 & 0.0948 & -0.2437 \\
\hline
\end{tabular}

Table 2 The Euler components of the plate motion 
Table 3 Comparison of the present-day spreading velocity at the mid-Atlantic ridge ${ }^{\text {a) }}$

\begin{tabular}{|c|c|c|c|c|c|c|c|c|c|}
\hline Latitude $/\left(^{\circ}\right)$ & Longitude $/\left(^{\circ}\right)$ & $V_{\mathrm{e}}(\mathrm{a})$ & $V_{\mathrm{n}}(\mathrm{a})$ & $V(a)$ & $V_{\mathrm{e}}(\mathrm{b})$ & $V_{\mathrm{n}}(\mathrm{b})$ & $V(\mathrm{~b})$ & $V(\mathrm{a})-V(\mathrm{~b})$ & $\Delta \alpha$ \\
\hline 70.0 & 344.60 & -14.7 & 5.2 & 15.6 & -16.3 & 5.3 & 17.1 & -1.5 & 1.5 \\
\hline 60.0 & 330.50 & -19.1 & 3.5 & 19.4 & -19.8 & 2.8 & 20.0 & -0.6 & 2.4 \\
\hline 51.2 & 330.00 & -21.7 & 3.5 & 21.9 & -21.6 & 2.7 & 21.8 & 0.1 & 2.1 \\
\hline 39.5 & 330.25 & -24.3 & 3.5 & 24.6 & -23.1 & 2.7 & 23.3 & 1.3 & 1.5 \\
\hline 38.4 & 329.70 & -21.6 & 1.8 & 21.7 & -19.2 & 4.8 & 19.8 & 1.9 & -9.2 \\
\hline 35.0 & 325.00 & -22.5 & 1.6 & 22.6 & -20.5 & 4.9 & 21.1 & 1.5 & -9.3 \\
\hline 30.9 & 318.30 & -23.5 & 1.4 & 23.5 & -21.9 & 5.1 & 22.5 & 1.0 & -9.7 \\
\hline 27.5 & 315.80 & -24.1 & 1.3 & 24.1 & -22.8 & 5.1 & 23.4 & 0.7 & -9.7 \\
\hline 24.5 & 313.90 & -24.6 & 1.2 & 24.6 & -23.5 & 5.1 & 24.0 & 0.6 & -9.4 \\
\hline 21.3 & 314.40 & -25.1 & 1.2 & 25.1 & -24.0 & 5.1 & 24.5 & 0.6 & -9.3 \\
\hline 20.3 & 314.30 & -21.4 & -0.6 & 21.4 & -23.2 & 1.7 & 23.3 & -1.9 & -5.8 \\
\hline 10.8 & 316.30 & -25.1 & -1.1 & 25.1 & -27.0 & 1.1 & 27.0 & -1.9 & -4.8 \\
\hline 0.50 & 335.00 & -28.3 & -5.9 & 28.9 & -30.4 & -4.0 & 30.7 & -1.8 & -5.3 \\
\hline-1.40 & 344.20 & -28.7 & -8.1 & 29.8 & -30.9 & -6.4 & 31.6 & -1.8 & -3.5 \\
\hline-10.5 & 347.00 & -30.2 & -8.7 & 31.4 & -32.6 & -7.1 & 33.4 & -2.0 & -3.8 \\
\hline-21.1 & 348.40 & -30.9 & -9.0 & 32.2 & -33.6 & -7.5 & 34.4 & -2.2 & -3.6 \\
\hline-31.1 & 346.60 & -30.7 & -8.6 & 31.9 & -33.5 & -7.0 & 34.2 & -2.3 & -3.8 \\
\hline-40.1 & 344.10 & -29.9 & -8.0 & 31.0 & -32.8 & -6.4 & 33.4 & -2.4 & -4.0 \\
\hline-48.2 & 350.20 & -27.8 & -9.3 & 29.3 & -30.7 & -7.9 & 31.7 & -2.4 & -4.1 \\
\hline-52.2 & 355.20 & -26.1 & -10.3 & 28.1 & -29.1 & -9.1 & 30.5 & -2.4 & -4.1 \\
\hline
\end{tabular}

a) $V_{\mathrm{e}}$ (a) and $V_{\mathrm{n}}$ (a) respectively represent the east and north rates of the mid-Atlantic ridge from ITRF2000VEL model; $V_{\mathrm{e}}$ (b) and $V_{\mathrm{n}}$ (b) respectively represent the east and north rates of the mid-Atlantic ridge from NNR-NUVEL1A model; $V(a)-V(b)$ and $\Delta \alpha$ are the dispersion of speed and of movement azimuth angle respectively.

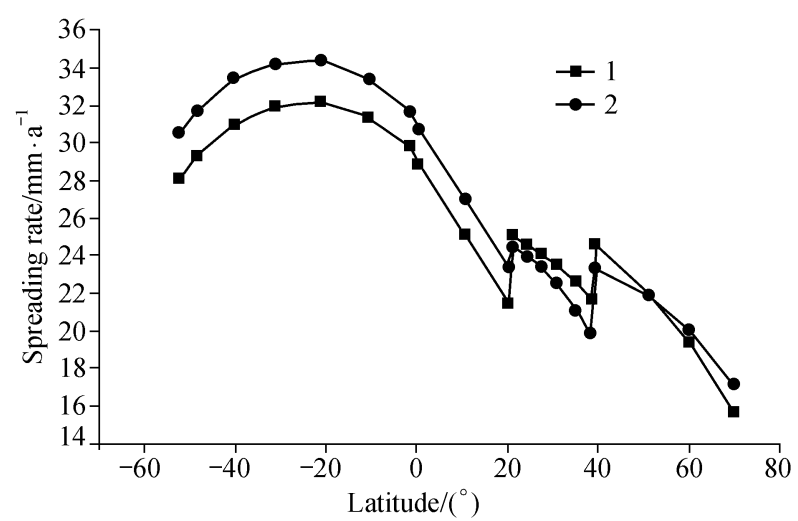

Fig. 1. Comparison of spreading rates. The solid square (1) represents the present-day spreading rate of the mid-Atlantic ridge obtained from space geodetic measurements, while the solid circle (2) represents the average spreading rate predicted by the geological and geomagnetic model NNR-NUVEL1A.

prediction here represents the 3-million-year average velocity. The global plate motion model of ITRF2000VEL is determined from an about 20-year time span of space geodetic data, so the model can better describe contemporary features of global plate motion. From table 1, the precise of angular velocity in ITRF2000VEL is average $0.002^{\circ} / \mathrm{Ma}$, and the precise of Euler pole is better than $1^{\circ}$. With respect to the NNR-NUVEL1A model, the maximal discrepancy is $0.02^{\circ} / \mathrm{Ma}$ in angular velocity and $12^{\circ}$ in Euler pole. The discrepancy is an order of magnitude larger than the precise of ITRF2000VEL, and even goes beyond the limits of errors in calculating Euler vector with space geodetic data. The difference between results from the NNR-NUVEL1A model and ITRF2000VEL model reflects the present-day motion characteristics of the mid-Atlantic ridge that the mid-ridge of the South Atlantic is slowing down, and the mid-ridge of the North Atlantic is speeding up.

Our latest studies record a slowdown of the spreading rate at the South mid-Atlantic ridge, mid-Indian ridge and also southeast mid-Pacific ridge, which may indicate a deceleration of the south hemisphere movement.

\section{Conclusion and discussion}

From the results above, spreading rates of the North, Equator and South mid-Atlantic ridge derived from ITRF2000VEL are $22.3 \mathrm{~mm} / \mathrm{a}, 28.8 \mathrm{~mm} / \mathrm{a}$ and $30.7 \mathrm{~mm} / \mathrm{a}$ respectively, basically aligning with the NNR-NUVEL1A predictions. It also shows that the spreading motion of the mid-Atlantic ridge in the recent 20 years is steady. Simultaneously the 3-million-year geological model 
NNR-NUVEL1A is proved to be correct. But the discrepancy between the two models is very significant for the contemporary crustal deformation measurement in $\mathrm{mm} / \mathrm{a}$ accuracy and just reflects current characteristics of the mid-Atlantic ridge spreading motion: the South mid-Atlantic ridge spreads faster than the North mid-Atlantic ridge while the former is slowing down and the latter is accelerating.

Provided that the mid-Atlantic ridge kept spreading at an average $25.6 \mathrm{~mm} / \mathrm{a}$ speed, it should have been around the Permian period or even earlier that the Atlantic Ocean came into being and began to spread. However, according to the geological data, the Atlantic Ocean did not exist in the Permian period. Therefore, we have reasons to suppose that during a certain period of geological time, the spreading rate of the mid-Atlantic ridge might be several times bigger than the current rate. Present-day movement characteristics of the mid-Atlantic ridge display a nonlinear spreading. As a result, we may conclude that the spreading of the mid-Atlantic ridge appears to be unsteady during different periods of time.

The plate motion and plate tectonics of those plates adjacent with the mid-Atlantic ridge have a very close relationship with the spreading of the mid-Atlantic ridge, especially for the South American plate and the African plate. With a westward strain from the South mid-Atlantic ridge and an eastward push from Nazca Plate collide, a transmeridional compressional deformation is seen on the South American plate as well as an integrated northward motion. While an eastward strain from the South midAtlantic ridge and a westward push from the East mid-Indian ridge force the whole African plate to move northeastward. Additionally, by analyzing the baseline change rates of those sites straddling the South Atlantic Ocean using data from velocity filed of sites distributed around the South American plate and the African plate, we obtain the baseline change rates varying in the range of 20 $-25 \mathrm{~mm} / \mathrm{a}$ for those sites of the same latitude degree, slower than the spreading rate of the South mid-Atlantic ridge by $5-10 \mathrm{~mm} / \mathrm{a}$, which may reflect the South American and African plates are not of rigid bodies.

It is of high significance to ascertain the correct spreading rate of the mid-Atlantic ridge so that we are able to better understand the theory of plate tectonics, the continental drifting theory and the environmental change. In our study, we are restricted by some uncertainties and factors: whether the African plate should be divided into two parts, the Nubia plate and somalia plate? the Eurasian plate sites concentrating in the West Europe, can the Euler's vector of the Eurasian Plate from the ITRF2000VEL model represent the whole Eurasia; sites on the South American Plate are rare and badly deformed by the strong push from the Nazca plate, etc. All these factors affect the accuracy of the space geodetic model. The higher-accuracy spreading rate depends on the further improvement in space techniques, a longer observation time span and a much wider and more average distributions of observation sites.

Acknowledgements This work was supported by the National Key Basic Research Project (Grant No. G1998040703), the Major Project for Basic Research of the Chinese Academy of Sciences (Grant No. KJ951-1-304) and the Scientific and Technological Development Foundation of Shanghai (Grant No. JC14012).

\section{References}

1. Ye, S., Kinetic Earth (in Chinese), Changsha: Hunan Science and Technology Press, 1996, 58-63.

2. Fu, C., 10th Lecture of the Earth (in Chinese), Beijing: Science Press, 1976, 102-159.

3. Shen, Z., Zhao, C., Yin, A. et al., Contemporary crustal deformation in East Asia constrained by Global Positioning System measurement, J. Gephys. Res., 2000, 105(B3): 5721.

4. Zhu, W., Wang, X., Cheng, Z. et al., Crustal Motion of Chinese Mainland Monitoring by GPS, Science in China, Series D, 2000 , 43(4): 394.

5. Sillard, P., Altamimi, Z., Boucher, C., The ITRF96 realization and its associated velocity field, Geophys. Res. Lett., 1998, 25(17): 3223.

6. Zhang, Q., Zhu, W., Xiong, Y., Global Plate Motion Models Incorporating the Velocity Field of ITRF96, Geophys. Res. Lett., 1999, 26(18): 2813.

7. Zhu, W., Han, J., Ma, W., Global plate motion model based on ITRF96 and ITRF97, Acta Astronomica Sinica, 2000, 41(3): 312.

8. Zhang, Q., Zhu, W., The initial establishment of the tectonic block motion model of China from space geodetic data, Chinese Science Bulletin, 2000, 45(9): 967.

9. DeMets, C., Gordon, R., Argus, D. et al., Effect of recent revisions to the geomagnetic reversal time scale on estimates of current plate motions, Geophys. Res. Lett., 1994, 21: 2191.

10. DeMets, C., Gordon, R., Argus, D. et al., Current plate motions, Geophys. J. Int., 1990, 101: 425.

(Received April 15, 2002) 